\title{
BMJ Open Quality Single-lead ECGs (AliveCor) are a feasible, cost-effective and safer alternative to 12-lead ECGs in community diagnosis and monitoring of atrial fibrillation
}

\author{
Jonathan James Hyett Bray (i) ,1,2 Elin Fflur Lloyd, ${ }^{3}$ Firdaus Adenwalla, ${ }^{4}$ \\ Sarah Kelly, ${ }^{4}$ Kathie Wareham, ${ }^{1,5}$ Julian P J Halcox ${ }^{1,6}$
}

To cite: Bray JJH, Lloyd EF, Adenwalla $F$, et al. Singlelead ECGs (AliveCor) are a feasible, cost-effective and safer alternative to 12-lead ECGs in community diagnosis and monitoring of atrial fibrillation. BMJ Open Quality 2021;10:e001270. doi:10.1136/ bmjoq-2020-001270

JJHB and EFL contributed equally.

Received 7 November 2020 Revised 20 February 2021 Accepted 4 March 2021

Check for updates

(C) Author(s) (or their employer(s)) 2021. Re-use permitted under CC BY-NC. No commercial re-use. See rights and permissions. Published by BMJ.

${ }^{1}$ Institute of Life Sciences 2, Swansea University Medical School, Swansea, UK

${ }^{2}$ General Medicine, Cardiff and Vale NHS Trust, Cardiff, UK

${ }^{3}$ Cardiology, Swansea Bay University Health Board, Port Talbot, UK

${ }^{4}$ Swansea Bay University Health Board, Port Talbot, UK

${ }^{5}$ Morriston Hospital, Swansea, UK

${ }^{6}$ Singleton Hospital, Swansea, Wales, UK

Correspondence to

Dr Jonathan James Hyett Bray; Jonathan.bray@swansea.ac.uk

\section{ABSTRACT}

Background Community management of atrial fibrillation (AF) often requires the use of electrocardiographic (ECG) investigation. Patients discharged following treatment of AF with fast ventricular response (fast AF) can require numerous ECGs to monitor rate and/or rhythm control. Single-lead ECGs have been proposed as a more convenient and relatively accurate alternative to 12-lead ECGs for rate/rhythm management and also diagnosis of AF. We aimed to examine the feasibility of using the AliveCor single-lead ECG monitor for diagnosis and monitoring of $\mathrm{AF}$ in the community setting.

Methods During the course of 6 months, this evaluation of a clinical service improvement pathway used the AliveCor in management of patients requiring (1) follow-up ECGs for AF with previously documented rapid ventricular rate or (2) ECG confirmation of rhythm where AF was suspected. Twelve AliveCor devices provided to the acute community medical team were used to produce 30 s ECG rhythm strips (iECG) that were electronically sent to an overreading physician.

Results Seventy-four patients (mean age 82 years) were managed on this pathway. (1) The AliveCor was successfully used to monitor the follow-up of 37 patients with fast $\mathrm{AF}$, acquiring a combined total of $113 \mathrm{iECGs}$ (median $1.5 \pm 3.75$ per patient). None of these patients required a subsequent 12-lead ECG and this approach saved an estimate of up to $£ 134.49$ per patient. (2) $0 f 53$ patients with abnormal pulses, the system helped identify 8 cases of new onset AF and 19 cases of previously known AF that had reverted from sinus back into AF.

Conclusions We have demonstrated that the AliveCor system is a feasible, cost-effective, time-efficient and potentially safer alternative to serial 12-lead ECGs for community monitoring and diagnosis of AF.

\section{INTRODUCTION}

Atrial fibrillation (AF) is a spectrum of disease and affects around $5.5 \%$ of adults aged 55 years and over. ${ }^{1}$ It can be either paroxysmal, persistent or permanent, and although some patients may be asymptomatic, it is often associated with symptoms

\section{Strengths and limitations}

- This is the first pilot initiative to demonstrate the feasibility of using a structured algorithm to facilitate the integration of single-lead ECGs into community monitoring and diagnosis of atrial fibrillation (AF).

- The AliveCor is US Food and Drug Administration approved and NICE evaluated, and therefore has the potential for wider integration into community AF management.

- The sample size is small, and the study is conducted in a single health board.

such as palpitations, breathlessness, fatigue and reduced exercise capacity. Patients with symptomatic AF are treated with either a rate or rhythm control strategy. Rate control is often the first-line approach for $\mathrm{AF}$ with an onset of greater than 48 hours, and can be achieved with acute and then long-term use of beta-blockers, calcium channel blockers, cardiac glycosides or combination therapy. Beta-blockers are considered first-line long-term therapy for patients with persistent or permanent $\mathrm{AF}$ and symptoms related to rapid ventricular rate (fast $\mathrm{AF}$ ) for symptomatic relief. Community follow-up monitoring is necessary to guide effective treatment. Conventionally, 12-lead ECGs have been used, but the advent of reliable single-lead ECGs with accurate built-in AF detection algorithms have the potential to streamline this monitoring process.

Single-lead ECGs could also have a role in community detection of AF. A diagnosis of AF can be difficult to make in the community as up to $62 \%$ is paroxysmal and up to $40 \%$ is asymptomatic. ${ }^{2}{ }^{3}$ In the UK, AF is detected through opportunistic investigation as systematic screening is currently 
not recommended by the UK National Screening Committee predominantly due to a lack of outcome evidence. ${ }^{4}$ Detection is important because new-onset AF is thought to account for one-fifth of patients hospitalised for ischaemic stroke. ${ }^{5}$ Indeed, AF is a well-established independent risk factor of ischaemic stroke, ${ }^{6}$ with convincing evidence for direct causality. ${ }^{5}$ Having controlled for confounding, device-detected AF lasting at least 1 hour in 3 months $(0.046 \%$ of the time) is associated with a 2.11-fold increase in stroke risk compared with no $\mathrm{AF}^{7}$ Anticoagulation in the form of direct oral anticoagulants or adjusted-dose warfarin reduces stroke risk by over $60 \%{ }^{8}$ NICE recommends anticoagulation in moderate- to high-risk patients. ${ }^{9}$

Previously, when ECG investigation had been required, our Acute Community Team in the Neath Port Talbot area have had to rely on undertaking a 12-lead ECG in the community. This can be a cumbersome and lengthy process that risks inappropriate tachyarrhythmia management, missed or delayed diagnosis of paroxysmal AF with adverse implications for initiation of appropriate anticoagulation. There is mounting evidence for the utility of integrating singlelead ECGs into healthcare provision in a number of settings. ${ }^{10-16}$ This pilot initiative aimed to test the feasibility of integrating a single-lead hand-held ECG system, the AliveCor, into community monitoring of treatment in patients with recently diagnosed fast $\mathrm{AF}$ and opportunistic community diagnosis of AF.

\section{METHOD}

\section{Participants and setting}

This evaluation of a clinical service improvement pathway enrolled a total of 74 patients from the Neath Port Talbot community between June and November 2017. Participants were eligible for inclusion if patients had been referred to the Acute Community Team with (1) known fast $\mathrm{AF}$ requiring monitoring and management, and (2) suspected AF due to an abnormal pulse on manual pulse check. There were no specific exclusion criteria. Baseline characteristics are recorded in table 1 .

\section{Patient and public involvement}

When asked, patients have expressed similar thoughts around the cumbersome nature of obtaining 12-lead ECG monitoring. Patients were involved at the stage of results collection. After providing informed verbal consent, patients were encouraged by the Acute Clinical Team to provide feedback on use of the AliveCor, which contributed to our results and discussion section.

\section{Single-lead ECG device (AliveCor-KardiaMobile)}

The AliveCor Kardia Mobile system (AliveCor, Mountain View, CA) consists of a battery-powered pair of electrodes and a Smartphone-based application linked using ultrasonic audio. ${ }^{17}$ The iECGs produced are a $30 \mathrm{~s}$ single-lead rhythm strip likened to lead 1 of a 12-lead ECG. iECGs can be input into accurate automatic algorithms (sensitivity $98 \%$, specificity $97 \%$ ) to detect $\mathrm{AF} .{ }^{18} \mathrm{It}$ is possible to store multiple iECGs and send them using WiFi securely to a physician. In this initiative, a local experienced academic clinical staff member trained the Acute Community Team members in using the AliveCor. Twelve AliveCor devices were provided to the community teams.

\section{Community atrial fibrillation monitoring}

Hospital discharges of newly treated AF with rapid ventricular response were referred to the Acute Community Team for ECG monitoring to ensure appropriate rate or rhythm control. Figure 1 shows how in the situation where a 12-lead ECG was not available, the AliveCor was used to direct optimised medical therapy. We recorded the number of iECGs required per patient and whether any further 12-lead ECG monitoring was required after use of the AliveCor (figure 2).

\section{Community atrial fibrillation diagnosis}

As demonstrated in figure 3, patients with symptoms consistent with $\mathrm{AF}$ were referred to the Acute Community Team for an initial clinical assessment. If the rhythm of the pulse was 'abnormal' or if the patient reported palpitations at the time of assessment, an ECG would be necessary. The AliveCor device was used if a 12-lead ECG was not immediately available. Had the use of the AliveCor not been possible, ambulatory Holter monitoring would have been arranged. We recorded the number of patients found to be in AF, and other rhythm disorders as interpreted by a cardiologist (table 2 ).

\section{Cost-effectiveness}

In 2019, NICE estimated the cost of a 12-lead ECG with either general practitioner (GP) or cardiologist overread to be between $£ 12.34$ and $£ 52^{19}$ and each AliveCor device to cost $£ 62.49+$ VAT. $^{20}$

Table 1 Patient characteristics

\begin{tabular}{llll}
\hline & Diagnosis & Rate control monitoring & Included participant overall average \\
\hline Participants $(\mathrm{n})$ & 53 & 37 & 90 \\
Mean age (years) & 78.7 & 79.8 & 81.6 \\
Sex (\% female) & 80 & 73 & 77
\end{tabular}

Patient characteristics for each group studied. The diagnosis group pertains to participants investigated for an abnormal pulse or symptoms consistent with $\mathrm{AF}$ and the monitoring group are participants followed up for optimal medical rate or rhythm control. 


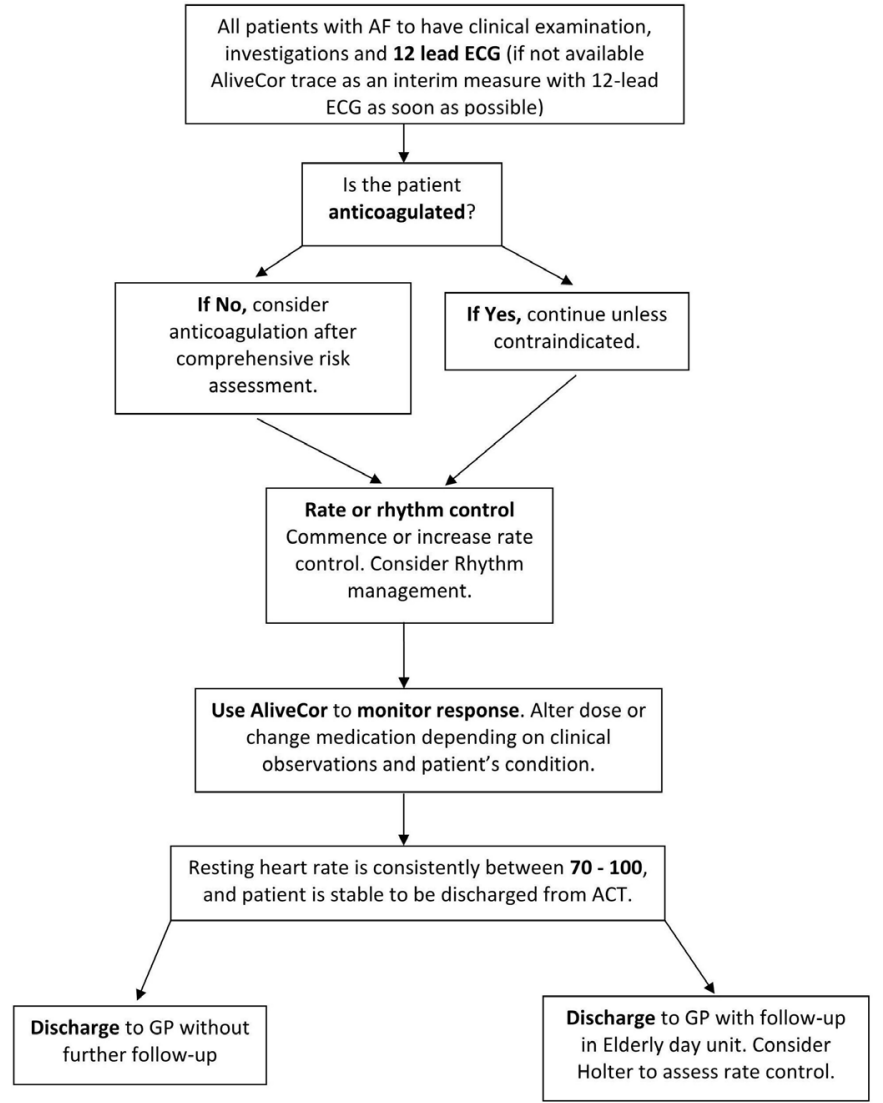

Figure 1 AF monitoring-patient flow for participants referred with fast $A F$ or whom develop fast $A F$ while under the care of the ACT. ACT, Acute Community Team; AF, atrial fibrillation; GP, general practitioner.

\section{Statistics}

Descriptive statistics were calculated and graphical data were presented using IBM SPSS V.26. OriginPro 2019b was used to construct the bar graph (figure 2).

\section{Ethical considerations}

According to WHO and Healthcare Quality Improvement Partnership guidance, risk-benefit analysis was performed internally. The proposal was deemed to be a

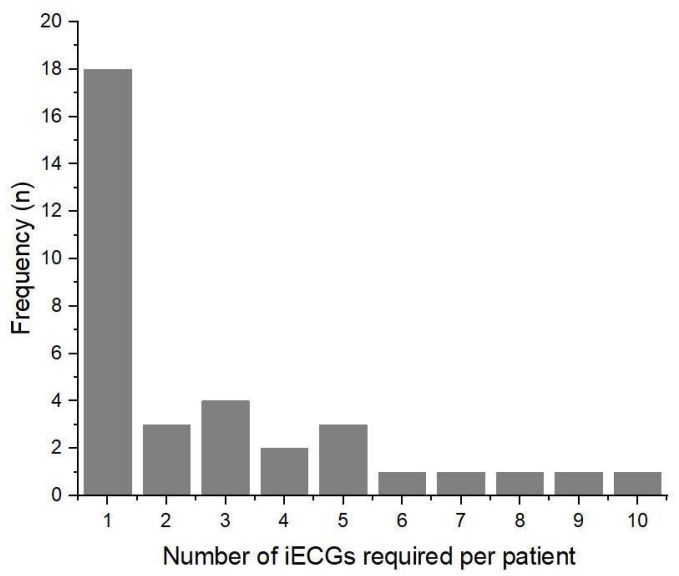

Figure 2 Histogram showing the number of iECGs per patient taken over a 6-month period. 'patient safety activity' as the AliveCor was used within a standard of care framework, the approach was tailored to improving provision of care in Swansea Bay University Health board, and the initiative did not involve vulnerable populations. The patient safety activity was considered of 'minimal risk' as it did not change treatment plans, data were not individually identifiable, the intervention did not divert staff from existing responsibilities and implementation did not constitute an elevated level of risk to patients or staff.

\section{RESULTS}

Baseline characteristics of the total 74 participants recruited ( $\mathrm{n}=37$ monitoring, $\mathrm{n}=53$ diagnosis) can be found in table 1. Participants were a mean age of 81.3 years and were predominantly female (female:male, 54:20). No participants were lost to follow-up.

\section{Community atrial fibrillation monitoring}

The 37 patients requiring ECG monitoring for follow-up of fast AF, required a total of 113 iECGs (median $1.5 \pm$ 3.75 per patient). The majority of patients only required one follow-up iECG to confirm adequate rate or rhythm control (figure 2). However, some patients required up to $10 \mathrm{iECGs}$ to titrate medication dose to appropriate clinical effect. There were no cases in which a 12-lead ECG was required due to the single-lead ECG not being sufficient. In terms of cost-effectiveness, assuming a 12-lead ECG would have been performed where in our initiative an iECG was required, the 12 AliveCor devices saved between $£ 495$ and $£ 4976$ according to NICE 2014 estimates, depending on GP or cardiologist overread. ${ }^{19} 20$ This equates to an average of $£ 13.37$ and $£ 134.49$ per patient requiring follow-up ECG monitoring.

\section{Community atrial fibrillation diagnosis}

Of the 53 patients assessed, 8 were found to have newonset AF, 19 patients with known AF (noted to be in sinus rhythm prior to index assessment) were found to have reverted back to AF, 7 had 'other' ECG abnormalities and 19 were normal (table 2). Of the eight patients found to have new onset $\mathrm{AF}$, five were female, with an average age of 78.3 years ( $\mathrm{SD} \pm 7.1$ years) and an average $\mathrm{CHA}_{2} \mathrm{DS}_{2}-$ VASc score of 4.9 ( $\mathrm{SD} \pm 0.85$, range $3-6$ ). All agreed to be appropriately anticoagulated. We found that as iECGs could be easily uploaded via a secure internet connection, they could be overread by a physician in an appropriately timely manner.

\section{DISCUSSION}

This single health board clinical service improvement pathway evaluation included 74 participants over a 6-month period using the US FDA-approved AliveCor. To our knowledge, this is the first pilot initiative to demonstrate the feasibility of using a structured algorithm to facilitate the integration of single-lead ECGs into community monitoring and diagnosis of $\mathrm{AF}$. We found that the 


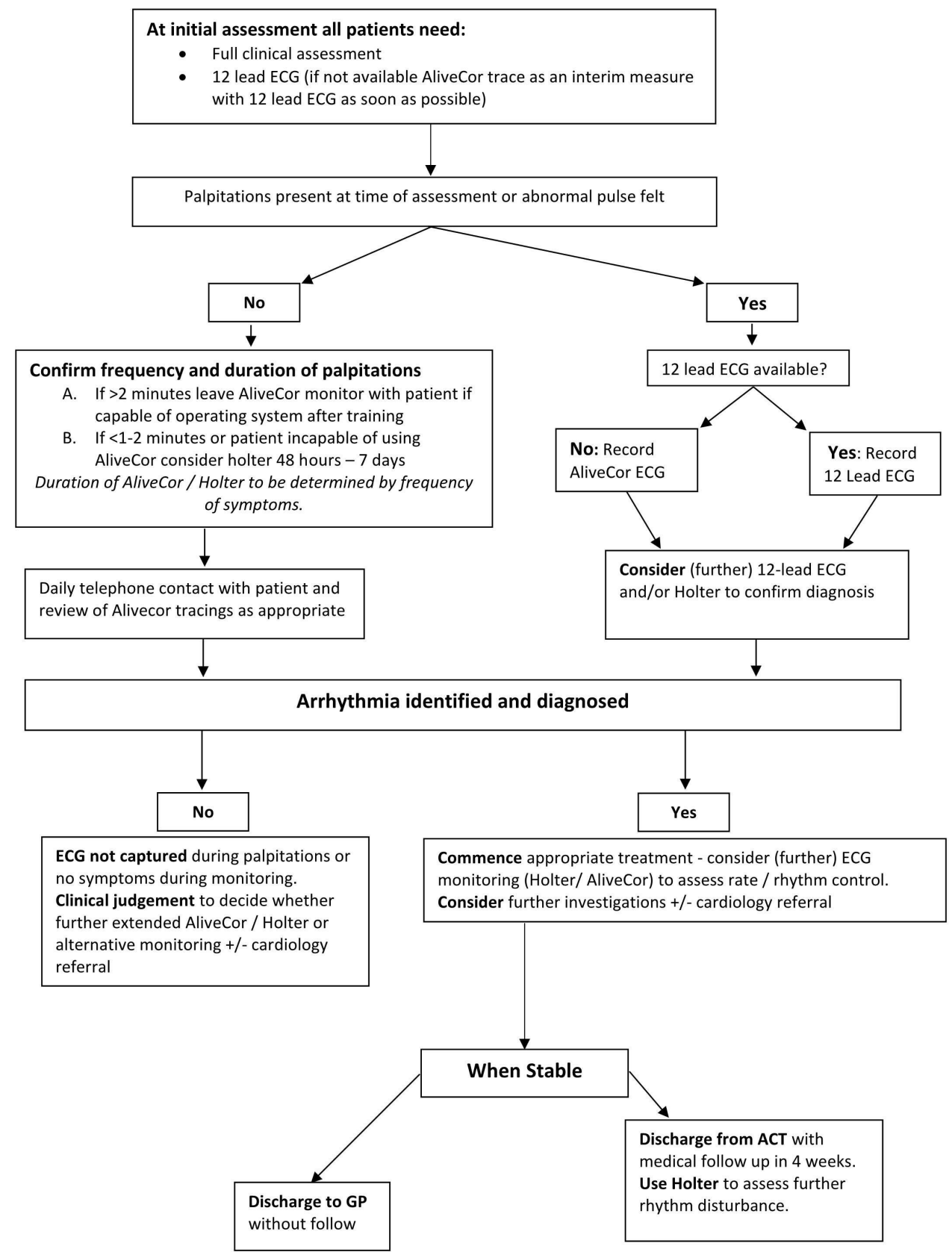

Figure 3 AF diagnosis - patient flow for patients with symptoms consistent with AF or an 'abnormal pulse' on palpation. ACT, Acute Community Team; AF, atrial fibrillation; GP, general practitioner.

AliveCor device was easy to use, more time-effective and cost-effective, and successfully prevented the need for serial 12-lead ECGs in the community. Of the 37 patients requiring ECG monitoring, 113 iECGs were needed and of the 53 patients with an 'abnormal' pulse, $15 \%$ were found to be in new-onset AF and were appropriately anticoagulated.

There are a number of commercially available portable ECG devices which are suitable for out-of-hospital use, ranging from one-lead to three-lead ECG devices. Portable ECGs have the advantage that they are easy to carry, relatively lightweight and low cost. The advantages of the AliveCor system is that it is (1) immediately accessible as an iECG, (2) portable, (3) user-friendly due to a filter for noise reduction and (4) is US Food and Drug Administration (FDA) approved and NICE evaluated. ${ }^{20}$ A recent meta-analysis by Duarte and colleagues examined the diagnostic accuracy of single-lead ECGs in four studies in asymptomatic individuals by a cardiologist or electrophysiologist and found a sensitivity of $93.9 \%(95 \%$ CI $86.2 \%$ to $97.4 \%$ ) and a specificity of $96.5 \%$ (95\% CI $90.4 \%$ to $98.8 \%$ ) for diagnosing $\mathrm{AF}^{21}$ which is considerably superior to manual pulse palpation (specificity $78.8 \%, 95 \%$ CI $51.0 \%$ to $94.5 \%){ }^{22}$

The AliveCor is the most widely studied single-lead ECG device, but there are other examples such as the Omron HeartScan ${ }^{23}$ or the Zenicor ECG ${ }^{24}$ which do not require smartphones. There are also wearable three-lead ECGs 
Table 2 Diagnoses using single-lead iECGs

\begin{tabular}{|c|c|c|c|c|c|}
\hline Month & Number & Normal & New AF & Known AF, found to be in AF & Other \\
\hline June & 9 & 5 & 1 & 2 & 1 tachycardia \\
\hline July & 9 & 3 & 0 & 4 & $\begin{array}{l}1 \text { tachycardia } \\
1 \text { atrial ectopy }\end{array}$ \\
\hline Aug & 5 & 0 & 1 & 2 & $\begin{array}{l}1 \text { bradycardia } \\
1 \text { tachycardia }\end{array}$ \\
\hline Oct & 12 & 7 & 2 & 3 & - \\
\hline Nov & 4 & 2 & 1 & 1 & - \\
\hline Total & 53 & 19 & 8 & 19 & 7 \\
\hline
\end{tabular}

Distribution of findings using the AliveCor device for patients with an abnormal pulse or symptoms of atrial fibrillation (AF).

such as the ZioPatch ${ }^{25}$ and the Nuvant mobile cardiac telemetry. ${ }^{26}$ Three-lead ECGs appear to be comparable with single-lead ECGs when assessing most intervals ${ }^{27}$; however, they do require longer application periods to obtain true readings and there can be a greater delay in accessing and reporting the ECG data. ${ }^{25}$

Integration of single-lead ECG devices into existing pathways has been demonstrated to be feasible in primary care,${ }^{10} 11$ opportunistically in pharmacies, ${ }^{12}{ }^{13}$ in lowresource settings, ${ }^{14}$ in rural areas ${ }^{15}$ and on a large scale. ${ }^{16}$ Similarly to our findings, all of these studies found a high degree of successful operator use. Six of these studies comment on uninterpretable traces and describe low rates with an average of $0.82 \%$ (SD $\pm 0.52 \%$, range $0 \%$ to $1.3 \%$ ). All of these studies report at least one training session as being sufficient for operators. In comparison with 12-lead ECGs, Holter monitoring and invasive event recorders, single-lead ECGs only require finger contact. Moreover, iECGs are designed to be transferred, analysed and stored. Once the application is set up, the system is relatively straightforward. Indeed, the vast majority of patients also appear to find the device easy to use. ${ }^{28}$

\section{Limitations and challenges}

To further evaluate the incorporation of single-lead ECGs into community $\mathrm{AF}$ diagnosis and monitoring pathways, this design can be extended to other health boards, increased in duration and participant number. Use of the AliveCor KardiaMobile was particularly valuable if there was a lack of time or if patients preferred not to undress for a 12-lead ECG. It was not difficult to obtain a trace with help, even in patients with poor dexterity or cognitive impairment. One of the main challenges encountered by the team was WiFi connectivity when out in the community on home visits. This meant that the clinical staff sometimes had to wait for a WiFi signal to resume before upload of iECGs was possible. With regard to documentation, particular care was taken to ensure traces were named. This was less of an issue with the online platform which comes with the AliveCor device (The Kardia Pro system). We collected verbal instead of written consent for inclusion, which limited our collection of patient data.

\section{Cost-effectiveness}

Another potential barrier to the integration of opportunistic use of single-lead ECGs in the National Health Service (NHS) is cost-effectiveness. The AliveCor appears to be a sustainable solution in the follow-up of AF with fast ventricular response as we saved an average of $£ 13.37$ to $£ 134.49$ per patient. Cost-effectiveness of single-lead ECGs have been demonstrated in similar circumstances. The multicentre, randomised trial $(n=243)$ by Reed and colleagues investigated the use of the AliveCor during symptoms over a 90-day period as opposed to ambulatory ECG monitoring. ${ }^{29}$ They found the AliveCor device saved $£ 474$ per patient compared with standard care. Moreover, Duarte and colleagues have estimated the costeffectiveness of using single-lead ECGs in a primary care setting compared with routine, opportunistic AF detection using manual pulse palpation. ${ }^{21}$

\section{Ongoing benefits}

We found that use of the AliveCor in the community cut down the number of patients needing to return to clinic. Moreover, the AliveCor is now used by our community team's clinicians in the field and also in clinic to quickly assess and confirm a patient's cardiac rhythm.

\section{CONCLUSION}

This initiative demonstrated the feasibility of integrating single-lead ECGs into NHS provision of community healthcare for patients with AF. We found that the AliveCor was a useful clinical adjunct to standard care as it provides an accurate, point-of-care elucidation of patient rhythm, saving valuable time and money in the delivery of acute community medical services.

Contributors JJHB is responsible for data formulation, data analysis, figure design, literature review, writing the original manuscript, editing the final manuscript and project administration. EFL is responsible for contributing to data formulation, figure design, contributing to writing the original manuscript and editing the final 
manuscript. FA contributed to running of the initiative and data collection. SK contributed to data collection. KW contributed to data formulation and was in charge of data collection and running of the initiative. JPJH conceptualised the initiative, reviewed the literature and made substantial contributions to editing of the manuscript.

Funding The authors have not declared a specific grant for this research from any funding agency in the public, commercial or not-for-profit sectors.

Competing interests None declared.

Patient and public involvement Patients and/or the public were not involved in the design, or conduct, or reporting, or dissemination plans of this research.

Patient consent for publication Not required.

Provenance and peer review Not commissioned; externally peer reviewed.

Data availability statement All data relevant to the study are included in the article.

Open access This is an open access article distributed in accordance with the Creative Commons Attribution Non Commercial (CC BY-NC 4.0) license, which permits others to distribute, remix, adapt, build upon this work non-commercially, and license their derivative works on different terms, provided the original work is properly cited, appropriate credit is given, any changes made indicated, and the use is non-commercial. See: http://creativecommons.org/licenses/by-nc/4.0/.

ORCID iD

Jonathan James Hyett Bray http://orcid.org/0000-0003-1167-6295

\section{REFERENCES}

1 Heeringa J, van der Kuip DAM, Hofman A, et al. Prevalence, incidence and lifetime risk of atrial fibrillation: the Rotterdam study. Eur Heart J 2006;27:949-53.

2 Brand FN, Abbott RD, Kannel WB, et al. Characteristics and prognosis of lone atrial fibrillation. 30-year follow-up in the Framingham study. JAMA 1985;254:3449-53.

3 Lip GY, Hee FL. Paroxysmal atrial fibrillation. QJM 2001;94:665-78.

4 The UK National Screening Committee. The UK NSC recommendation on atrial fibrillation screening in adults. Available: https://legacyscreening.phe.org.uk/atrialfibrillation2019 [Accessed 1 Apr 2020].

5 Jaakkola J, Mustonen P, Kiviniemi T, et al. Stroke as the first manifestation of atrial fibrillation. PLoS One 2016;11:9.

6 Wolf PA, Abbott RD, Kannel WB. Atrial fibrillation as an independent risk factor for stroke: the Framingham study. Stroke 1991;22:983-8.

7 Boriani G, Glotzer TV, Santini M, et al. Device-detected atrial fibrillation and risk for stroke: an analysis of $>10,000$ patients from the SOS AF project (Stroke preventiOn Strategies based on Atrial Fibrillation information from implanted devices). Eur Heart $J$ 2014;35:508-16.

8 Granger CB, Armaganijan LV. Newer oral anticoagulants should be used as first-line agents to prevent thromboembolism in patients with atrial fibrillation and risk factors for stroke or thromboembolism. Circulation 2012;125:159-64.

9 National Institute for Health and Care Excellence (NICE). Atrial Fibrillation - Management. Available: https://cks.nice.org.uk/atrialfibrillation\#!scenario2019 [Accessed 1 Apr 2020].

10 Grubb NR, Elder D, Broadhurst P, et al. Atrial fibrillation case finding in over $65 \mathrm{~s}$ with cardiovascular risk factors - results of initial Scottish clinical experience. Int J Cardiol 2019;288:94-9.

11 Himmelreich JCL, Karregat EPM, Lucassen WAM, et al. Diagnostic accuracy of a smartphone-operated, single-lead electrocardiography device for detection of rhythm and conduction abnormalities in primary care. Ann Fam Med 2019;17:403-11.
12 Lowres N, Neubeck L, Salkeld G, et al. Feasibility and costeffectiveness of stroke prevention through community screening for atrial fibrillation using iPhone ECG in pharmacies. Thromb Haemost 2014;111:1167-76.

13 Staszewsky L, Pasina L, Musazzi UM, et al. Screening for unknown atrial fibrillation in older people: a feasibility study in community pharmacies. Eur Geriatr Med 2018;9:113-5.

14 Evans GF, Shirk A, Muturi P, et al. Feasibility of using mobile ECG recording technology to detect atrial fibrillation in low-resource settings. Glob Heart 2017;12:285-9.

15 Chen $\mathrm{Y}-\mathrm{H}$, Hung $\mathrm{C}-\mathrm{S}$, Huang $\mathrm{C}-\mathrm{C}$, et al. Atrial fibrillation screening in nonmetropolitan areas using a telehealth surveillance system with an embedded cloud-computing algorithm: prospective pilot study. JMIR Mhealth Uhealth 2017;5:e135.

16 Chan N-yin, Choy C-chung, Choy CC. Screening for atrial fibrillation in 13122 Hong Kong citizens with smartphone electrocardiogram. Heart 2017;103:24-31.

17 KardiaMobile. Alivecor information, 2020. Available: https://www. kardiamobile.co.uk/kardiamobile [Accessed 1 Apr 2020].

18 Lau JK, Lowres N, Neubeck L, et al. iPhone ECG application for community screening to detect silent atrial fibrillation: a novel technology to prevent stroke. Int J Cardiol 2013;165:193-4.

19 National Institute for Health and Care Excellence (NICE). Lead-I ECG devices for detecting symptomatic atrial fibrillation using single time point testing in primary care. Available: https://www.nice.org.uk/ guidance/dg35/chapter/4-Evidence [Accessed 26 Jun 2020].

20 National Institute for Health and Care Excellence (NICE). AliveCor heart monitor and AliveECG APP (Kardia mobile) for detecting atrial fibrillation. Available: https://www.nice.org.uk/advice/mib35/chapter/ Summary [Accessed 26 Jun 2020].

21 Duarte R, Stainthorpe A, Greenhalgh J, et al. Lead-I ECG for detecting atrial fibrillation in patients with an irregular pulse using single time point testing: a systematic review and economic evaluation. Health Technol Assess 2020;24:1-164.

22 Welton NJ, McAleenan A, Thom HH, et al. Screening strategies for atrial fibrillation: a systematic review and cost-effectiveness analysis. Health Technol Assess 2017;21:1-236.

23 de Asmundis C, Conte G, Sieira J, et al. Comparison of the patient-activated event recording system vs. traditional $24 \mathrm{~h}$ Holter electrocardiography in individuals with paroxysmal palpitations or dizziness. Europace 2014;16:1231-5.

24 Usadel L, Haverkämper G, Herrmann S, et al. Arrhythmia detection in pediatric patients: ECG quality and diagnostic yield of a patienttriggered Einthoven Lead-I event recorder (Zenicor EKG-2 ${ }^{\mathrm{TM}}$ ). Pediatr Cardiol 2016;37:491-6.

25 Barrett PM, Komatireddy R, Haaser S, et al. Comparison of 24-hour Holter monitoring with 14-day novel adhesive patch electrocardiographic monitoring. Am J Med 2014;127:95.e11-95. e17.

26 Engel JM, Mehta V, Fogoros R. Study of arrhythmia prevalence in NUVANT mobile cardiac telemetry system patients. 34th annual International Conference of the IEEE Engineering-in-Medicine-andBiology-Society (EMBS). San Diego, CA. New York: leee, 2012.

27 Brockway R, Brockway M, Brockway B, et al. Comparison of oneand three-lead ECG to measure cardiac intervals and differentiate drug-induced multi-channel block. J Pharmacol Toxicol Methods 2018;93:80-9.

28 Halcox JPJ, Wareham K, Cardew A, et al. Assessment of remote heart rhythm sampling using the AliveCor heart monitor to screen for atrial fibrillation: the REHEARSE-AF study. Circulation 2017;136:1784-94.

29 Reed MJ, Grubb NR, Lang CC, et al. Multi-centre randomised controlled trial of a smart phone-based event recorder alongside standard care versus standard care for patients presenting to the emergency department with palpitations and pre-syncope - the IPED (Investigation of Palpitations in the ED) study: study protocol for a randomised controlled trial. Trials 2018;19:9. 